Article

\title{
Nucleotide Sequence Variation in Long-Term Tissue Cultures of Chinese Ginseng (Panax ginseng C. A. Mey.)
}

\author{
Sitong Liu ${ }^{1}$, Xinfeng Wang ${ }^{2}$, Ning Ding ${ }^{2}$, Yutong Liu ${ }^{3}$, Ning $\mathrm{Li}^{3}{ }^{\circledR}$, Yiqiao Ma ${ }^{4}$, Jing Zhao ${ }^{3}$, Zhenhui Wang ${ }^{5}$, \\ Xiaomeng $\mathrm{Li}^{3}$, Xueqi Fu ${ }^{1, *}$ and Linfeng $\mathrm{Li}^{2, *}$ \\ 1 School of Life Sciences, Jilin University, Changchun 130012, China; liust17@mails.jlu.edu.cn \\ 2 Ministry of Education Key Laboratory for Biodiversity Science and Ecological Engineering, \\ School of Life Sciences, Fudan University, Shanghai 200438, China; wangxinfeng@fudan.edu.cn (X.W.); \\ 14307110009@fudan.edu.cn (N.D.) \\ 3 Key Laboratory of Molecular Epigenetics of Ministry of Education (MOE), Northeast Normal University, \\ Changchun 130024, China; liuyt718@nenu.edu.cn (Y.L.); lin690@nenu.edu.cn (N.L.); \\ zhaoj878@nenu.edu.cn (J.Z.); lixm441@nenu.edu.cn (X.L.) \\ 4 Jilin Academy of Vegetable and Flower Sciences, Changchun 130033, China; xiaoquezai@163.com \\ 5 Department of Agronomy, Jilin Agricultural University, Changchun 130118, China; wzhjlau@163.com \\ * Correspondence: fxq@jlu.edu.cn (X.F.); lilinfeng@fudan.edu.cn (L.L.)
}

Citation: Liu, S.; Wang, X.; Ding, N.; Liu, Y.; Li, N.; Ma, Y.; Zhao, J.; Wang, Z.; Li, X.; Fu, X.; et al. Nucleotide Sequence Variation in Long-Term Tissue Cultures of Chinese Ginseng (Panax ginseng C. A. Mey.). Plants 2022, 11, 79. https://doi.org/ $10.3390 /$ plants 11010079

Academic Editor: Yoshihisa Ikeda

Received: 26 November 2021

Accepted: 24 December 2021

Published: 27 December 2021

Publisher's Note: MDPI stays neutral with regard to jurisdictional claims in published maps and institutional affiliations.

Copyright: (C) 2021 by the authors. Licensee MDPI, Basel, Switzerland. This article is an open access article distributed under the terms and conditions of the Creative Commons Attribution (CC BY) license (https:// creativecommons.org/licenses/by/ $4.0 /$ )

\begin{abstract}
Plants have the salient biological property of totipotency, i.e., the capacity to regenerate a whole plant from virtually any kind of fully differentiated somatic cells after a process of dedifferentiation. This property has been well-documented by successful plant regeneration from tissue cultures of diverse plant species. However, the accumulation of somaclonal variation, especially karyotype alteration, during the tissue culture process compromises cell totipotency. In this respect, Chinese ginseng (Panax ginseng C. A. Mey.) is an exception in that it shows little decline in cell totipotency accompanied by remarkable chromosomal stability even after prolonged tissue cultures. However, it remains unclear whether chromosomal level stability necessarily couples with molecular genetic stability at the nucleotide sequence level, given that the two types of stabilities are generated by largely distinct mechanisms. Here, we addressed this issue by genome-wide comparisons at the single-base resolution of long-term tissue culture-regenerated $P$. ginseng plants. We identified abundant single nucleotide polymorphisms (SNPs) that have accumulated in cultured ginseng callus and are retained in the process of plant regeneration. These SNPs did not occur at random but showed differences among chromosomes and biased regional aggregation along a given chromosome. In addition, our results demonstrate that, compared with the overall genes, genes related to processes of cell totipotency and chromosomal stability possess lower mutation rates at both coding and flanking regions. In addition, collectively, the mutated genes exhibited higher expression levels than non-mutated genes and are significantly enriched in fundamental biological processes, including cellular component organization, development, and reproduction. These attributes suggest that the precipitated molecular level genetic variations during the process of regeneration in $P$. ginseng are likely under selection to fortify normal development. As such, they likely did not undermine chromosomal stability and totipotency of the long-term ginseng cultures.
\end{abstract}

Keywords: cell totipotency; chromosome stability; nucleotide sequence variation; whole genome resequencing; tissue culture; Panax ginseng

\section{Introduction}

Plants are sessile by nature and have evolved sophisticated developmental and phenotypic plasticity to adapt to new and changing environments [1]. Perhaps one of the most remarkable aspects of this adaptation is totipotency, referring to the ability of fully differentiated plant somatic cells to regenerate to an intact plant after a process of dedifferentiation [2-5]. Over the past decades, cell totipotency of cultured plant cells has been 
widely documented in diverse plant species [6-8]. Nevertheless, cell totipotency of cultured plant cells often declines rapidly with time in culture and often losses the trait completely within 1-2 years [9]. Mechanisms underlying this phenomenon are not well understood, but one suspected culprit is the disruption of normal cellular controls during culture, which leads to the occurrence and accumulation of genome instability, especially chromosomal instability (CIN) [10,11].

CIN includes two major aspects: numerical and structural. Numerical CIN refers to loss and/or gain of whole chromosomes or chromosomal segments, while structural CIN refers to translocation, inversion, and microscopically visible segmental gain and/or loss [12]. Conceivably CIN, being saltational changes in karyotype, may impair any biological properties especially complex ones (such as cell totipotency) that entail intricate regulation by many genes at the pathway and network levels [11]. It is, therefore, not surprising that the occurrence and accumulation of CINs would undermine totipotency or abrogate it altogether. By contrast, most molecular level mutations, such as single nucleotide polymorphisms (SNPs), are neutral, given that only a small percentage of the nucleus DNA of any higher eukaryotic organism is protein-coding genes, and even in which there is the mechanism of codon degeneracy or substantial differences in functional importance along with an open reading frame. Moreover, the two types of genome stabilities, chromosomal and molecular, are known to be controlled by distinct mechanisms $[13,14]$. Together, it is reasonable to assume that CIN is not necessarily coupled with molecular level genetic instability, and the later, if impacts totipotency at all, should do so at a much lower probability.

Chinese ginseng (Panax ginseng C.A. Meyer) is a perennial herb species within the genus Panax L. of family Araliaceae $[15,16]$. Molecular phylogeny and evolutionary history inferences have revealed a recent whole genome duplication (WGD) that occurred some 2-3 million years ago, which led to the formation of an ancestral tetraploid $(2 \mathrm{n}=4 x=48)$ followed by the splitting into three sister species, namely P. ginseng, P. japonicus and P. quinquefolius $[17,18]$. In East Asia, Chinese ginseng is widely used as a traditional herbal medicine to allegedly restore stamina and enhance the capacity to cope with fatigue and physical stress for $>2000$ years $[19,20]$. Recent studies of Chinese ginseng have also illustrated its pharmacological effects on treatment of some cancers, diabetes, cardiovascular disease, and central nervous dysfunction [21,22].

Apart from its medicinal and medical importance, $P$. ginseng also has several salient biological properties. One of these that we recently discovered [23] is the maintenance of totipotency over prolonged tissue cultures, which is accompanied by high chromosomal stability - both features are unknown in any other plant. Specifically, we found that Chinese ginseng callus subcultured for 12 consecutive years remained chromosomally stable and showed little decline in cell totipotency [23]. Further transcriptomic analyses suggested that sustained high expression of genes related to tissue totipotency and chromosomal stability might be associated with the manifestation of these two traits [23]. Nevertheless, several previous studies have shown that ginseng tissue cultures manifest mutations at the nucleotide sequence level. For example, Kiselev et al. (2013) analyzed four functionally important genes in two- and 20-y-old ginseng cultures and found that all four genes showed nucleotide variation that accumulates with culture duration [24,25]. These studies, albeit based on only a few pre-selected genes, suggest that in an aspect of molecular level genetic variation under tissue culture, ginseng is perhaps not much different from other plants. Nonetheless, to have a definitive answer to this matter, genome-wide analyses are needed, which are currently lacking.

Here, we addressed this issue based on findings reported in our previous study [23]. Specifically, we performed whole-genome resequencing and conducted genome-wide, single base-resolution analyses for molecular level genetic variations in plants regenerated from long-term cultured calli that were still highly totipotent and showed little CIN [23]. We characterized the SNPs with respect to their relevance to genomic features, chromosomal distribution, gene category, and expression. We conclude that molecular level genetic 
variation occurred in prolonged tissue cultures of $P$. ginseng, and which were retained in regenerated plants. However, these molecular level mutations may not have affected chromosomal stability and totipotency.

\section{Methods and Materials}

\subsection{Plant Materials and Callus Culture}

The original ginseng explant was taken from a single bud of $P$. ginseng cv. Damaya in 2004 [23]. The primary callus was induced with a standard Murashige-Skoog (MS) solid medium containing $2 \mathrm{mg} / \mathrm{L} 2,4$-dichlorophenoxyacetic acid (2,4-D) at $26{ }^{\circ} \mathrm{C}$ under dark conditions for 1 month. Then, the newly formed embryogenic calli were selected and subcultured with MS medium under the same conditions (at 30-day intervals) for 12-13 years [23]. The embryogenic calli remained totipotent, and in 2016 and 2017, they were transferred to a regeneration medium containing MS basal $+0.3 \%$ hydrolyzed casein acid $+0.1 \%$ proline $+1 \mathrm{mg} / \mathrm{L} 2,4-\mathrm{D}$ and culturing at $26^{\circ} \mathrm{C}$ under $14 / 10$ light/dark conditions [26]. The regenerated ginseng plantlets were taken for subsequent experiments.

\subsection{DNA Extraction and Whole Genome Resequencing}

The first batch of samples included 20 randomly selected ginseng plantlets regenerated from 12-y-old ginseng calli, and which were arbitrarily divided into two pools. The second patch of the sample included seven regenerated plantlets from 13-y-old ginseng calli. DNA extraction of the three pooled samples were conducted using a modified CTAB method [27]. The high-quality genomic DNA of each sample was utilized for subsequent whole-genome resequencing. Short pair-end $(150 \mathrm{bp})$ DNA libraries of the three samples were constructed with Illumina Trueseq DNA PCR-Free kit at Novogene (Tianjin, China) and sequenced with the Illumina Hi-seq 4000 platform (Illumina, CA, US). The data were deposited in the SRA database of GenBank (http:/ / www.ncbi.nlm.nih.gov/sra, accessed on 20 November 2021) with the BioProject accession number PRJNA782439. Only clean reads (base quality > 30) were retained for subsequent data analyses.

\subsection{Identification of De Novo Genetic Variants and Their Chromosomal Distribution}

To identify genetic variants accumulated in these tissue culture-regenerated plant samples, clean short reads of the three pooled samples were mapped to the $P$. ginseng reference genome using Burrows-Wheeler Aligner (BWA) [28], with default parameters. Then, the program Samtools [29], Genome Analysis Toolkit (GATK) [30] and Picard (http:/ / broadinstitute.github.io/picard/, accessed on 19 November 2021) were used to sort, index, and realign the mapped short reads, and remove duplicates. To identify SNPs with high confidence, the realigned short reads were further filtered using the program Bcftools [29], with the parameters "-a DP,ADF,ADR -Q 30 -q 30 -Ou”. Low-quality SNPs with base quality $(\mathrm{Q})$ or mapping quality $(\mathrm{q})$ smaller than 30 were excluded from the data analyses. In addition, we performed additional quality control of the SNPs according to the following procedures: (1) each allele of the heterozygous (Ht) SNPs possessing $>$ five mapped reads; (2) the homozygous (Ho) SNPs with read depth $\geq 5$ were retained; (3) SNPs with multi-allelic $(\geq 3)$ variants, insertions and deletions were excluded. Only those SNPs that passed the above quality controls were used for further analyses. Based on these identified Ht SNPs, we further defined the de novo variants as those that were non-shared among the three samples (y_2016_1,y_2016_2 and y_2017), which should be variations that occurred during tissue culture and independently retained in the regenerated plants.

\subsection{Functional Analyses of Mutated Genes}

The program SnpEff [31] was used to annotate the identified SNPs from the three pooled samples independently. Then, we calculated the number of non-synonymous (Sn) and synonymous (Ss) mutations for each gene. Based on this annotated information, all genes were divided into two categories, namely mutated gene (containing SNPs) and non-mutated gene (without SNPs). The mutated gene was further divided into two types, 
Sn gene (containing Sn mutations) and the other type (no Sn mutations). Functions of genes in different classes were analyzed and inferred using WEGO 2.0 (Web Gene Ontology Annotation Plot, http://wego.genomics.org.cn/, accessed on 21 November 2021) [32]. Gene expression data and genomic pattern of genes in different classes were retrieved from our previous study [23]. Distribution patterns of the identified SNPs were visualized using R package "ggplots" and "RColorBrewer". Correlation between gene mutation and expression level was analyzed in R package "ggplots".

\section{Results}

\subsection{Identification of Molecular Level Genetic Mutations in Ginseng Plantlets Regenerated from Long-Term Callus}

Whole-genome resequencing of the three pooled ginseng callus-regenerated plantlet samples generated a total of 1,638,664,104 clean pair-end Illumina reads (reads length $=150 \mathrm{bp}$ ), with each sample possessing 36-58x genome coverage (Table S1). Based on this deep sequencing data, we identified a total of 1,480,353 Ht SNPs from the three samples, each containing from 336,972 to $1,137,450$. Among these nucleotide variants, 343,330 were common to all three samples (Table 1). Given that (1) the ginseng plants are known as genetically heterogeneous among individuals even within the same cultivar (e.g., Damaya), and (2) we no longer had the exact plant individual (as the control) from which the explant was taken to initiate the callus cultures, we assigned SNPs shared by all three samples as standing genetic differences between our explant individual and the ginseng reference genome. As such, only the 1,137,023 Ht SNPs that are non-shared among the three samples were defined as de novo variants that occurred during the callus culture process and were retained in the regenerated plantlets (Table 1 ). Although, to an extent, this conservative treatment may lead to an underestimate of the culture-induced variations, it confidently ruled out false positives. Albeit potential underestimated, our data clearly show that somaclonal variation in the form of SNPs definitively occurred in the long-term cultured ginseng callus.

Next, we interrogated how these de novo genetic variants were associated with genomic features in the ginseng genome. At the whole genome level, the y_2017 sample $(0.34 \mathrm{Ht} / \mathrm{Kb})$ contained higher nucleotide diversity compared to both of the two y_2016 samples $(0.18$ and $0.10 \mathrm{Ht} / \mathrm{Kb})$. At the genic region, all three samples showed slightly lower nucleotide diversity $(0.09-0.26 \mathrm{Ht} / \mathrm{Kb})$ relative to the whole genome level. Notably, the cell totipotency- $(0.06-0.16 \mathrm{Ht} / \mathrm{Kb})$ and chromosome stability-related genes possessed obviously lower nucleotide diversity at genic regions compared to the genome-wide overall genic regions. We also calculated the number of Ss and Sn mutations in the three samples (Table S2). A general pattern is that all three samples contained more Sn than Ss mutations for the overall and cell totipotency/chromosome stability-related genes. Among the three samples, the y_2017 sample (Ss = 8666 and Sn = 16,806) harbored higher numbers of Ss and Sn mutations compared to the two y_2016 callus samples (Ss = 3851-6699 and $\mathrm{Sn}=7271-12,691)$ for the overall genes. Likewise, we also identified smaller numbers of $\mathrm{Ss}$ and $\mathrm{Sn}$ mutations in the cell totipotency-related genes ( $\mathrm{Ss}=3.95-15.30 \times 10^{-6}$ and $\left.\mathrm{Sn}=9.37-25.20 \times 10^{-6}\right)$ and chromosome stability-related genes $\left(\mathrm{Ss}=8.62-24.80 \times 10^{-6}\right.$ and $\left.S n=13.90-45.60 \times 10^{-6}\right)$ than in those of the overall genes $\left(S s=12.10-27.10 \times 10^{-6}\right.$ and $\left.\mathrm{Sn}=22.80-52.60 \times 10^{-6}\right)$.

\subsection{Spectrum and Trend of the Molecular Level Genetic Mutations}

The above analyses revealed distinct levels of nucleotide diversity among the three ginseng callus-regenerated plantlet samples and differences in different genomic regions. We then calculated the spectrum of the genetic variants along the 24 ginseng chromosomes (Figure 1). Results showed that these genetic variants were not randomly distributed along a given ginseng chromosome, and the amount of mutations differed among the chromosomes. For example, each of the 24 chromosomes possessed several high-mutation genomic regions (referred to as mutation clusters) across the three samples. In addition, some chromosomes 
(i.e., Chr06) harbored more mutation clusters than the others (i.e., Chr24). In particular, all the homeologous chromosome pairs of the two subgenomes differed in the number and distribution pattern of mutation clusters. Broadly, all three samples showed similar distribution patterns of mutation clusters along the 24 chromosomes.

Table 1. The number of heterozygous (Ht) genetic variants and nucleotide diversity in three pooled callus-regenerated plantlets of Panax ginseng.

\begin{tabular}{|c|c|c|c|c|c|c|}
\hline Items & y_2016_1 & y_2016_2 & y_2017 & Total Ht SNPs * & $\begin{array}{l}\text { Ht SNPs Shared } \\
\text { by All Three } \\
\text { Samples }\end{array}$ & $\begin{array}{l}\text { De Novo Ht SNPs in } \\
\text { the Three Samples \& }\end{array}$ \\
\hline $\begin{array}{l}\text { Genome-wide } \\
\text { heterozygotes }\end{array}$ & 591,204 & 336,972 & $1,137,450$ & $1,480,353$ & 343,330 & $1,137,023$ \\
\hline Genome size (bp) & $3.36 \times 10^{9}$ & $3.36 \times 10^{9}$ & $3.36 \times 10^{9}$ & $3.36 \times 10^{9}$ & NA & $3.36 \times 10^{9}$ \\
\hline $\begin{array}{c}\text { Average genome-wide } \\
\mathrm{Ht}(\text { per } \mathrm{Kb})\end{array}$ & 0.18 & 0.10 & 0.34 & 0.44 & NA & 0.34 \\
\hline Genic heterozygotes & 55,308 & 28,718 & 83,996 & 118,949 & 27,347 & 91,602 \\
\hline Length of all genes (bp) & $3.20 \times 10^{8}$ & $3.20 \times 10^{8}$ & $3.20 \times 10^{8}$ & $3.20 \times 10^{8}$ & NA & $3.20 \times 10^{8}$ \\
\hline Genic Ht (per Kb) & 0.17 & 0.09 & 0.26 & 0.37 & NA & 0.29 \\
\hline $\begin{array}{l}\text { Totipotency-related } \\
\text { genic heterozygotes }\end{array}$ & 174 & 114 & 329 & 450 & 98 & 352 \\
\hline $\begin{array}{l}\text { Length of all totipotency- } \\
\text { related genes }(\mathrm{bp})\end{array}$ & $2.03 \times 10^{6}$ & $2.03 \times 10^{6}$ & $2.03 \times 10^{6}$ & $2.03 \times 10^{6}$ & NA & $2.03 \times 10^{6}$ \\
\hline $\begin{array}{l}\text { Totipotency-related } \\
\text { genic } \mathrm{Ht}(\text { per } \mathrm{Kb})\end{array}$ & 0.09 & 0.06 & 0.16 & 0.22 & NA & 0.17 \\
\hline $\begin{array}{c}\text { Chromosomal stability- } \\
\text { Related genic heterozygotes }\end{array}$ & 563 & 244 & 875 & 1272 & 257 & 1015 \\
\hline $\begin{array}{l}\text { Length of all chromosomal } \\
\text { stability- genes (bp) }\end{array}$ & $3.94 \times 10^{6}$ & $3.94 \times 10^{6}$ & $3.94 \times 10^{6}$ & $3.94 \times 10^{6}$ & NA & $3.94 \times 10^{6}$ \\
\hline $\begin{array}{l}\text { Chromosomal stability- } \\
\text { related genic Ht (per Kb) }\end{array}$ & 0.14 & 0.06 & 0.22 & 0.32 & NA & 0.26 \\
\hline
\end{tabular}

We next estimated the spectrum of Ss and Sn mutations separately along each chromosome of the three samples (Figure S1). Similar to the mutation spectrum at the overall level, both Ss and Sn variants also exhibited distinct variation patterns across the 24 chromosomes. For example, both the number and distribution of mutation clusters differed between the Ss and Sn mutations within and between the 24 chromosomes. In addition, our results also revealed that the mutation clusters trended towards the telemetric regions (Figure S1). However, an opposite distribution pattern was observed for the overall genetic variants (see Figure 1). It is notable that while the three samples showed a highly similar overall distribution pattern of these genetic variants, they possessed some sample-specific mutation clusters of all the three types of genetic variants (overall, Ss and Sn), especially those on the centromeric region of Chr06.

\subsection{Associations between Genetic Variation and Gene Expression}

The above analyses revealed clustered distribution of the genetic variants. We next examined whether the mutated genes would show different transcription levels compared to the non-mutated (wild-type) genes. A general pattern observed at the overall level was that genes localized at distal (subtelomeric) regions showed higher expression levels compared to those of at the interstitial (pericentromeric) regions, although each of the 24 chromosomes showed different overall transcription levels (Figure 2a). Between the two subgenomes, the 12 homeologous chromosome pairs possessed highly similar expression pattern at the distal regions. For example, genes localized at the two distal genomic regions 
of the homologous chromosome pair 1 and 9 showed distinct transcription levels. Further analysis of the relationship between gene transcription and genetic variants, we found that the mutated genes possessed higher transcription levels than those of non-mutated (i.e., wild-type) genes in all three samples (Figure 2b). Furthermore, mutated genes containing non-synonymous mutations showed relatively higher expression levels compared to genes with synonymous mutations, although both exhibited higher transcription levels relative to the non-mutated genes.

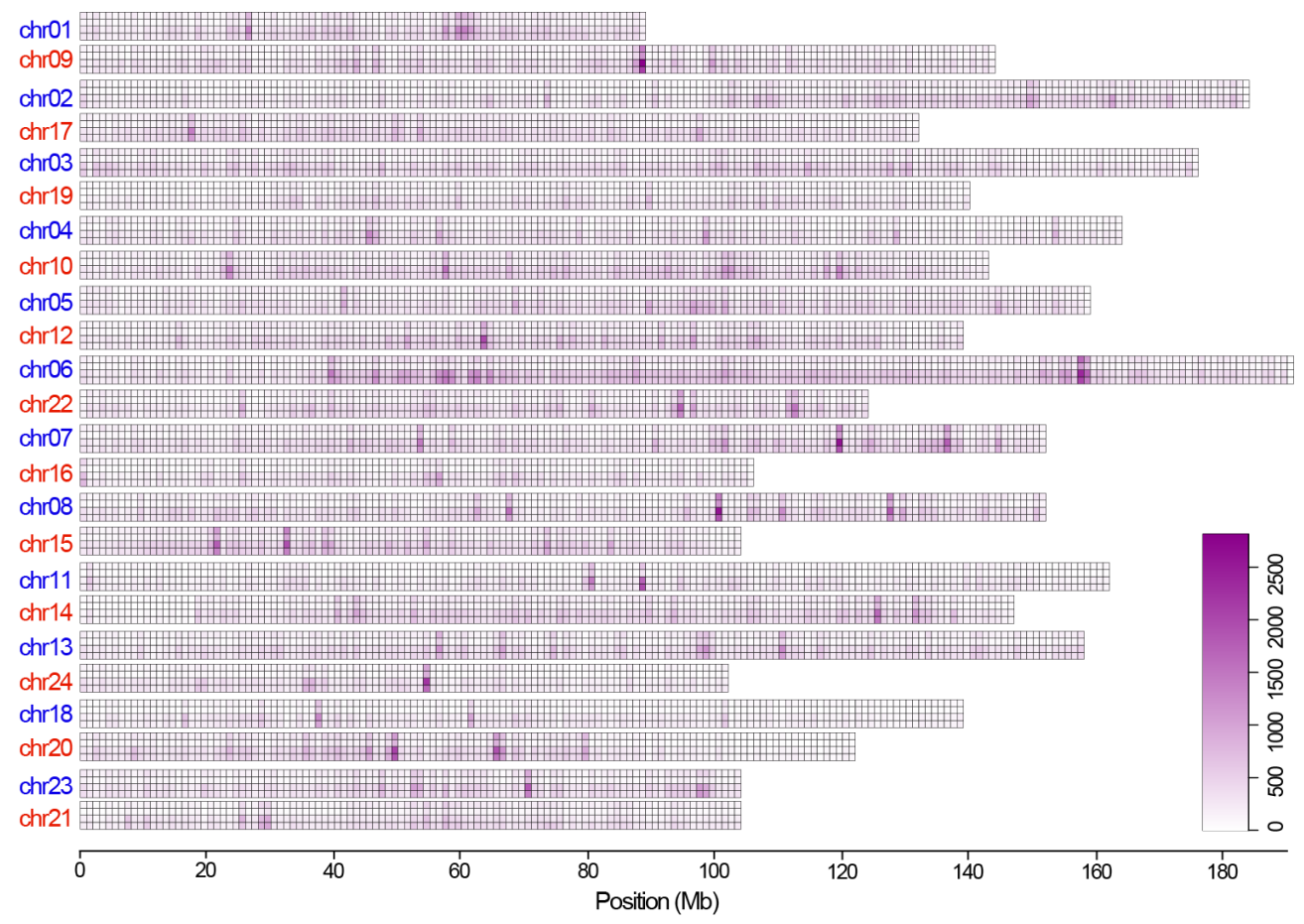

Figure 1. Genomic/chromosomal landscape of the genetic variants identified in the three pooled ginseng callus-regenerated plantlet samples. The four rows from top to bottom for each chromosome are genetic variants identified in samples y_2016_1,y_2016_2,y_2017, and de novo genetic variants. The numbers of the four types of Ht SNPs are the same as in Table 1. Homeologous chromosomes of the two ginseng subgenomes are labeled in blue and red, respectively.
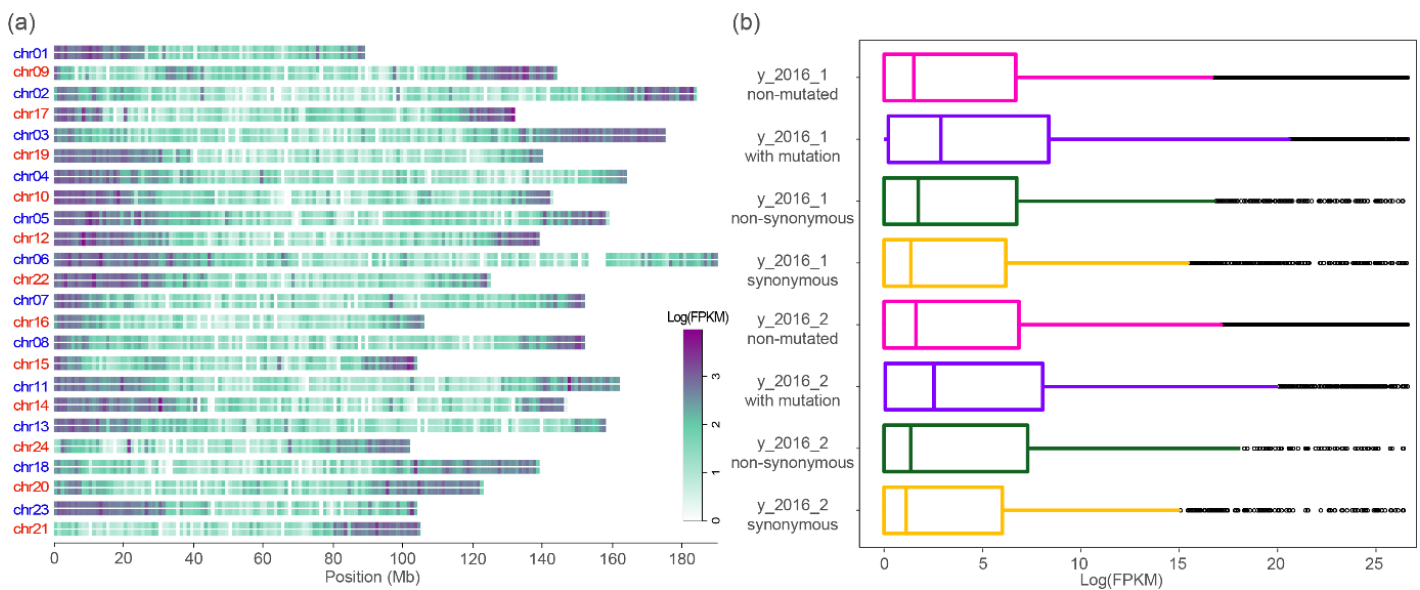

Figure 2. Genome-wide gene expression pattern and correlation with a genetic mutation. (a) The transcription level of the total genes along each of the 24 chromosomes. The two rows for each chromosome correspond to the sample y_2016_1 and y_2016_2, respectively; (b) comparisons between the overall gene expression levels and the types of genetic variants. 


\subsection{Functional Enrichment of the Mutated Genes}

All genetic variants identified in the three regenerated plantlet samples should have occurred during the callus subculture process. We then asked the question: were these mutated genes functionally associated with chromosome stability and cell totipotency? Functional enrichment analyses revealed that the cell totipotency- and chromosome stabilityrelated genes showed distinct enrichment patterns compared to the total genes (Figure 3). At the cellular component level, for example, compared to the cell totipotency-related genes and total genes, the chromosome stability-related genes showed relatively higher enrichment in the gene ontology (GO) terms related to intracellular cell, organelle part, and supramolecular complex. A similar pattern was also observed at the molecular function level where the chromosome stability-related genes exhibited higher enrichment in protein activity, molecular and chemical compound binding. At the biological process, however, the cell totipotency-related genes exhibited higher percentages of enrichment in these GO terms involved in diverse metabolic processes, plant development, and stress responses.

Based on the above functional analyses at the overall level, we further examined whether a similar enrichment pattern existed in the mutated genes. Our results revealed that mutated genes of the three samples showed different GO term enrichments compared to the overall genes, as detailed above (Figures S2-S4). In the cellular component category, the mutated genes of sample y_2016_1 exhibited a similar enrichment pattern to the overall genes, with the majority of the enriched GO terms related to intracellular cell and organelle part. However, the other two samples (y_2016_2 and y_2017) were enriched to the other cellular components, such as the membrane part and supramolecular complex. In contrast, the three samples exhibited moderate differences in the functional enrichments in the molecular function and biological process categories. For example, compared to the nonmutated genes, mutated genes of the three samples showed functional enrichment in biological processes related to metabolic process, development, reproduction, and stress responses. In the molecular function category, the mutated genes were mainly enriched in functions related to protein activity and molecular binding.

It is notable that the mutated and non-mutated genes showed different functional enrichments. In the cellular component category, the chromosome stability-related genes exhibited a higher degree of enrichment in components related to intracellular, organelle and supramolecular complex (Figures S2-S4). However, the mutated genes containing Ss and $\mathrm{Sn}$ mutations exhibited lower degrees of functional enrichment than the non-mutated genes. Yet, the mutated genes possessed similar enriched GO terms to the cell totipotencyand chromosome stability-related genes in the biological process and molecular function categories. The synonymous and non-synonymous genes exhibited higher degrees of enrichment than the non-mutated genes, particularly those related to cellular component organization, plant development, and reproduction. In addition, we also examined the mutation types for the cell totipotency- and chromosome stability-related genes. A general pattern was that majority of these important functional genes possessed multiple copies in the ginseng genome (Table S3). For example, 108 of the 388 cell totipotency-related genes were functionally related to auxin efflux carrier and response in the ginseng genome (Table S4). Likewise, we also identified 88 casein kinase1 (CK1) and 102 CGMC (CGMC includes CDA, MAPK, GSK3, and CLKC kinases) in the 533 chromosome stability-related genes (Table S5). It is notable that, among the 388 cell totipotency-related genes, some functionally important genes possessed both the $\mathrm{Sn}$ and Ss mutations, including those involved in auxin response and DNA methylation. Similarly, we also identified Sn and Ss mutations in some of the 533 chromosome stability-related genes, such as dnaK, minichromosome maintenance subunit 2/3(MCM2/3), and CK1. 
(a)

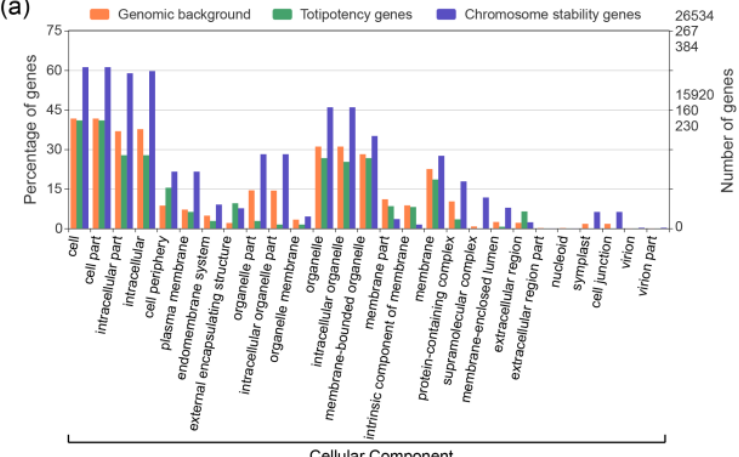

(d)

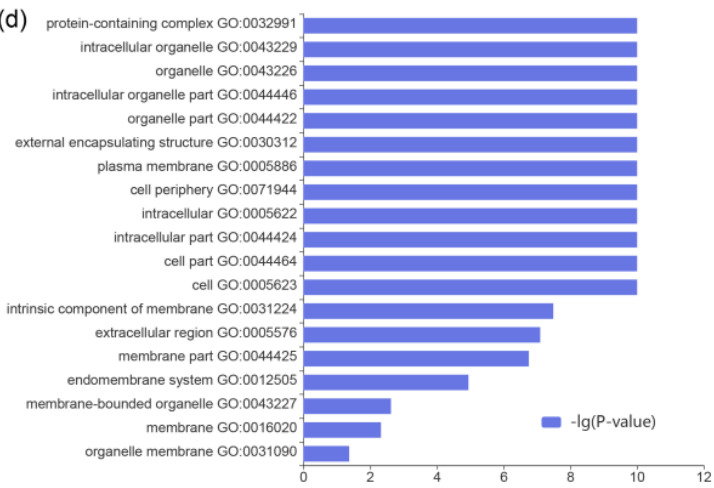

$35379 \quad(\mathrm{e})$

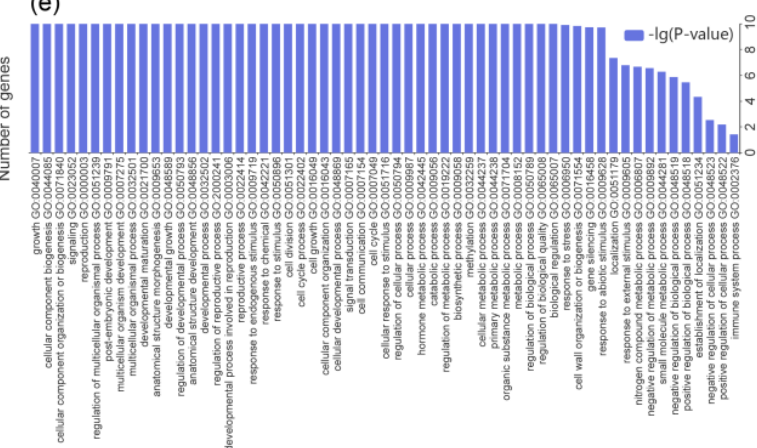

(f)

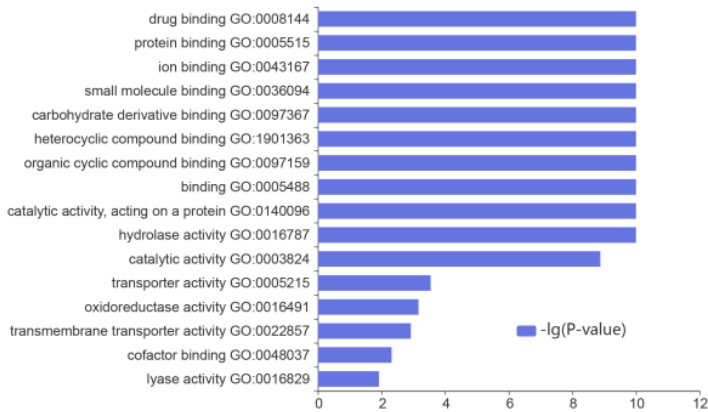

Molecular Function

Figure 3. GO enrichment analysis of the total totipotency and chromosome stability-related genes. Enriched GO terms in cellular component $(\mathbf{a}, \mathbf{d})$, biological process $(\mathbf{b}, \mathbf{e})$ and molecular function of the total genes $(\mathbf{c}, \mathbf{f})$, totipotency genes and chromosome stability genes.

\section{Discussion}

Plants possess a generally stronger capacity to regenerate new tissues and organs upon loss or injury of body parts under natural conditions than animals [33-35]. Plant in vitro tissue culture experiments have uncovered the even more remarkable property of plants, i.e., totipotency, referring to the phenomenon that fully differentiated plant somatic cells are capable of re-differentiating into intact plants after a de-differentiation phase [36-39]. However, during tissue culture, diverse genetic and epigenetic instabilities (collectively termed somaclonal variation [10]) occur, which on the one hand may comprise the elite phenotypes of the donor plants while on the other hand can be exploited for beneficial variants $[9,13]$. While tissue culture-induced epigenetic variation mainly involves changes in DNA methylation, that is, loss/gain of methylated cytosine bases [40], genetic variation is more complex and can be broadly categorized into chromosomal- and sequence-level mutations [41]. It is generally believed that somaclonal variation is a major cause of the rapid decline and/or loss of totipotency in plant tissue cultures [42]. 
We reported recently that, different from other plant species, a cultured ginseng somaclonal callus line showed little decline of totipotency even after $>12$ years in subculture [23]. Of note, our study showed that this ginseng somaclonal callus line maintains high chromosomal stability in both number and structure. As far as we know, these two properties of $P$. ginseng have not been observed in any animals (with respect to somatic chromosomal stability) and other plants. However, previous studies have shown that mutations at the nucleotide sequence level occurred in ginseng tissue cultures, with mutation rate increases in a time-dependent fashion [24,25]. It thus seems that ginseng was not particularly different from other plants in this aspect, which appears incongruent with our results [23]. However, chromosomal and nucleotide sequence instabilities are known to be controlled by distinct pathways $[13,14,43]$. Therefore, it is possible that sequence-level mutations also occurred in the callus somaclonal line we studied [23], which did not undermine totipotency. If this were the case, it also would be interesting to learn what features these mutations have.

Here, we investigated this matter based on genome-wide resequencing and analyses of ginseng plantlets regenerated from the same somatic callus line that showed remarkable chromosomal stability [23]. We uncovered numerous genetic variants at the nucleotide sequence level, although we have adopted a highly conserved treatment of the data, i.e., excluding the variants that are shared by all three samples, each containing a pool of regenerated plantlets (see Results). We found several features of these mutations, including (i) emergence of mutation clusters and their biased distribution towards distal chromosome regions; (ii) higher non-synonymous than synonymous mutation rates; (iii) lower mutation rates in cell totipotency- and chromosomal stability-related genes than overall genes; (iv) higher expression levels in mutated genes than non-mutated genes; and (v) enrichment of mutated genes to key basic biological processes including cellular component organization, development, and reproduction.

Notably, because we analyzed regenerated plantlets rather than callus per se, the mutations we detected should be a small fraction of those that occurred in the callus, and, which were retained in the regeneration process. Thus, there is no doubt that mutations at the nucleotide sequence level did occur in cultured ginseng cells, consistent with prior studies $[24,25]$. The features we summarized above may suggest that mutations in our long-term ginseng cultures did not occur at random. Moreover, they were likely under both purifying and positive selections. First, because distal chromosome regions are known as recombination-active, if somatic recombination events [44] had occurred in the long-term culture process, then it is possible that deleterious mutations can be purged out. Second, both higher non-synonymous than synonymous mutation rates and higher expression levels of mutated than non-mutated genes suggest the possibility of selection. Third, lower than average mutation rates in genes related to cell totipotency and chromosomal stability suggest either non-random occurrence of mutation or more efficient repairing for these critical genes. Finally, specific enrichment of mutated genes to fundamental biological processes may suggest their potential roles to compensatory for maintaining critical cellular homeostasis and to fortify normal development. Together, these features of the mutations we uncovered in the long-term ginseng cultures strongly suggest that they did not undermine chromosomal stability nor compromise cell totipotency. For example, several genes related to auxin response, i.e., auxin response factor 19 and auxin efflux carrier component, possess numerous non-synonymous mutations. It has been documented that genes involved in auxin and cytokinin biosynthesis play important roles in the cell totipotency of cultured plant cells [1,34]. This suggests that these genetic mutations are potentially associated with the maintaining of cell totipotency in the long-term tissue culture process.

Supplementary Materials: The following supporting information can be downloaded at: https: / / www.mdpi.com/article/10.3390/plants11010079/s1, Figure S1. Genomic landscape of the nonsynonymous (a) and synonymous (b) mutations. The four rows for each chromosome correspond to the samples y_2016_1, y_2016_2, y_2017, and de novo genetic variants. Homologous chromosomes 
of the two subgenomes were labeled in blue and red, respectively. Figure S2. GO enrichment analysis of the mutated genes in the sample y_2016_1. Figure S3. GO enrichment analysis of the mutated genes in the sample y_2016_2. Figure S4. GO enrichment analysis of mutated genes in the sample y_2017. Table S1. Basic information of the raw data in the three ginseng callus-regenerated plantlet samples. Table S2. Summary of the non-synonymous and synonymous mutations in the three ginseng callus-regenerated plantlet samples. Table S3. Totipotency- and chromosome stability-related genes in the ginseng genome. Table S4. Mutation type of the totipotency-related genes. Table S5. Mutation type of the chromosome stability-related genes.

Author Contributions: Conceptualization, X.F. and L.L.; formal analysis, S.L. and X.W.; funding acquisition, X.F., X.L. and L.L.; investigation, S.L., X.W., N.D., Y.L., N.L. and Z.W.; resources, Y.L., N.L., J.Z. and Y.M.; supervision, X.F. and X.L.; writing-original draft, S.L. and X.W.; writing-review and editing, X.F. and L.L. All authors have read and agreed to the published version of the manuscript.

Funding: This research was supported by the National Natural Science Foundation of China (NSFC31970235) to L.L.

Data Availability Statement: All Illumina data generated in this study has been deposited to SRA under the accession number of PRJNA782439.

Conflicts of Interest: The authors declare no conflict of interest.

\section{References}

1. Su, Y.H.; Tang, L.P.; Zhao, X.Y.; Zhang, X.S. Plant cell totipotency: Insights into cellular reprogramming. J. Integr. Plant Biol. 2021, 63, 228-240. [CrossRef]

2. Koltunow, A.M.; Grossniklaus, U. Apomixis: A developmental perspective. Annu. Rev. Plant Biol. 2003, 54, 547-574. [CrossRef]

3. Steward, F.C.; Mapes, M.O.; Mears, K. Growth and Organized Development of Cultured Cells. II. Organization in Cultures Grown from Freely Suspended Cells. Am. J. Bot. 1958, 45, 705-708. [CrossRef]

4. Haberlandt, G. Culturversuche mit isolierten Pflanzenzellen. Sitz. Kais. Akad. Wiss. Math.-Nat. Cl. 1902, 61, 1-23.

5. Feher, A. Somatic embryogenesis-Stress-induced remodeling of plant cell fate. Biochim. Biophys. Acta 2015, 1849, 385-402. [CrossRef] [PubMed]

6. Green, C.E.; Phillips, R.L. Plant Regeneration from Tissue Cultures of Maize1. Crop Sci. 1975, 15, 417-421. [CrossRef]

7. Heyser, J.W.; Dykes, T.A.; DeMott, K.J.; Nabors, M.W. High frequency, long term regeneration of rice from callus culture. Plant Sci. Lett. 1983, 29, 175-182. [CrossRef]

8. Sugimoto, K.; Jiao, Y.; Meyerowitz, E.M. Arabidopsis regeneration from multiple tissues occurs via a root development pathway. Dev. Cell 2010, 18, 463-471. [CrossRef] [PubMed]

9. Neelakandan, A.K.; Wang, K. Recent progress in the understanding of tissue culture-induced genome level changes in plants and potential applications. Plant Cell Rep. 2012, 31, 597-620. [CrossRef]

10. Larkin, P.J.; Scowcroft, W.R. Somaclonal variation-A novel source of variability from cell cultures for plant improvement. Theor. Appl. Genet. 1981, 60, 197-214. [CrossRef]

11. Phillips, R.L.; Kaeppler, S.M.; Olhoft, P. Genetic Instability of Plant-Tissue Cultures-Breakdown of Normal Controls. Proc. Natl. Acad. Sci. USA 1994, 91, 5222-5226. [CrossRef]

12. Tang, Y.C.; Amon, A. Gene copy-number alterations: A cost-benefit analysis. Cell 2013, 152, 394-405. [CrossRef] [PubMed]

13. Fossi, M.; Amundson, K.; Kuppu, S.; Britt, A.; Comai, L. Regeneration of Solanum tuberosum Plants from Protoplasts Induces Widespread Genome Instability. Plant Physiol. 2019, 180, 78-86. [CrossRef] [PubMed]

14. Lee, J.K.; Choi, Y.L.; Kwon, M.; Park, P.J. Mechanisms and Consequences of Cancer Genome Instability: Lessons from Genome Sequencing Studies. Annu. Rev. Pathol. 2016, 11, 283-312. [CrossRef] [PubMed]

15. Lee, C.; Wen, J. Phylogeny of Panax using chloroplast trnC-trnD intergenic region and the utility of trnC-trnD in interspecific studies of plants. Mol. Phylogenet. Evol. 2004, 31, 894-903. [CrossRef]

16. Zuo, Y.J.; Wen, J.; Zhou, S.L. Intercontinental and intracontinental biogeography of the eastern Asian-Eastern North American disjunct Panax (the ginseng genus, Araliaceae), emphasizing its diversification processes in eastern Asia. Mol. Phylogenet. Evol. 2017, 117, 60-74. [CrossRef]

17. Choi, H.I.; Kim, N.H.; Lee, J.; Choi, B.S.; Kim, K.D.; Park, J.Y.; Lee, S.C.; Yang, T.J. Evolutionary relationship of Panax ginseng and P. quinquefolius inferred from sequencing and comparative analysis of expressed sequence tags. Genet. Resour. Crop Evol. 2013, 60, 1377-1387. [CrossRef]

18. Shi, F.X.; Li, M.R.; Li, Y.L.; Jiang, P.; Zhang, C.; Pan, Y.Z.; Liu, B.; Xiao, H.X.; Li, L.F. The impacts of polyploidy, geographic and ecological isolations on the diversification of Panax (Araliaceae). BMC Plant Biol. 2015, 15, 297. [CrossRef]

19. Gillis, C.N. Panax ginseng pharmacology: A nitric oxide link? Biochem. Pharmacol. 1997, 54, 1-8. [CrossRef]

20. Hemmerly, T.E. A Ginseng farm in Lawrence County, Tennessee. Econ. Bot. 1977, 31, 160-162. [CrossRef] 
21. Li, C.; Zhu, Y.; Guo, X.; Sun, C.; Luo, H.; Song, J.; Li, Y.; Wang, L.; Qian, J.; Chen, S. Transcriptome analysis reveals ginsenosides biosynthetic genes, microRNAs and simple sequence repeats in Panax ginseng C. A. Meyer. BMC Genom. 2013, 14, 245. [CrossRef] [PubMed]

22. Wee, J.J.; Mee Park, K.; Chung, A.S. Biological Activities of Ginseng and Its Application to Human Health. In Herbal Medicine: Biomolecular and Clinical Aspects; Benzie, I.F.F., Wachtel-Galor, S., Eds.; CRC Press: Boca Raton, FL, USA, 2011.

23. Liu, S.; Zhao, J.; Liu, Y.; Li, N.; Wang, Z.; Wang, X.; Liu, X.; Jiang, L.; Liu, B.; Fu, X.; et al. High Chromosomal Stability and Immortalized Totipotency Characterize Long-Term Tissue Cultures of Chinese Ginseng (Panax ginseng). Genes 2021, $12,514$. [CrossRef]

24. Kiselev, K.V.; Dubrovina, A.S.; Shumakova, O.A. DNA mutagenesis in 2- and 20-yr-old Panax ginseng cell cultures. In Vitro Cell. Dev. Biol. Plant 2013, 49, 175-182. [CrossRef]

25. Kiselev, K.V.; Shumakova, O.A.; Tchernoded, G.K. Mutation of Panax ginseng genes during long-term cultivation of ginseng cell cultures. J. Plant Physiol. 2011, 168, 1280-1285. [CrossRef] [PubMed]

26. Zhang, D.; Wang, Z.H.; Wang, N.N.; Gao, Y.; Liu, Y.; Wu, Y.; Bai, Y.; Zhang, Z.B.; Lin, X.Y.; Dong, Y.Z.; et al. Tissue Culture-Induced Heritable Genomic Variation in Rice, and Their Phenotypic Implications. PLoS ONE 2014, 9, e96879. [CrossRef] [PubMed]

27. Kidwell, K.K.; Osborn, T.C. Simple plant DNA isolation procedures. In Plant Genomes: Methods for Genetic and Physical Mapping; Beckmann, J.S., Osborn, T.C., Eds.; Springer: Dordrecht, The Netherlands, 1992; pp. 1-13.

28. Li, H. Aligning sequence reads, clone sequences and assembly contigs with BWA-MEM. arXiv 2013, arXiv:1303.3997.

29. Li, H.; Handsaker, B.; Wysoker, A.; Fennell, T.; Ruan, J.; Homer, N.; Marth, G.; Abecasis, G.; Durbin, R.; Genome Project Data Processing Subgroup. The Sequence Alignment/Map format and SAMtools. Bioinformatics 2009, 25, 2078-2079. [CrossRef]

30. McKenna, A.; Hanna, M.; Banks, E.; Sivachenko, A.; Cibulskis, K.; Kernytsky, A.; Garimella, K.; Altshuler, D.; Gabriel, S.; Daly, M.; et al. The Genome Analysis Toolkit: A MapReduce framework for analyzing next-generation DNA sequencing data. Genome Res. 2010, 20, 1297-1303. [CrossRef]

31. Cingolani, P.; Platts, A.; Wang, L.L.; Coon, M.; Nguyen, T.; Wang, L.; Land, S.J.; Lu, X.; Ruden, D.M. A program for annotating and predicting the effects of single nucleotide polymorphisms, SnpEff: SNPs in the genome of Drosophila melanogaster strain $\mathrm{w}^{1118}$; iso-2; iso-3. Fly 2012, 6, 80-92. [CrossRef]

32. Ye, J.; Zhang, Y.; Cui, H.; Liu, J.; Wu, Y.; Cheng, Y.; Xu, H.; Huang, X.; Li, S.; Zhou, A.; et al. WEGO 2.0: A web tool for analyzing and plotting GO annotations, 2018 update. Nucleic Acids Res. 2018, 46, W71-W75. [CrossRef]

33. Birnbaum, K.D.; Sanchez Alvarado, A. Slicing across kingdoms: Regeneration in plants and animals. Cell 2008, 132, 697-710. [CrossRef]

34. Ikeuchi, M.; Favero, D.S.; Sakamoto, Y.; Iwase, A.; Coleman, D.; Rymen, B.; Sugimoto, K. Molecular Mechanisms of Plant Regeneration. Annu. Rev. Plant Biol. 2019, 70, 377-406. [CrossRef]

35. Ikeuchi, M.; Ogawa, Y.; Iwase, A.; Sugimoto, K. Plant regeneration: Cellular origins and molecular mechanisms. Development 2016, 143, 1442-1451. [CrossRef] [PubMed]

36. Hu, B.; Zhang, G.; Liu, W.; Shi, J.; Wang, H.; Qi, M.; Li, J.; Qin, P.; Ruan, Y.; Huang, H.; et al. Divergent regeneration-competent cells adopt a common mechanism for callus initiation in angiosperms. Regeneration 2017, 4, 132-139. [CrossRef] [PubMed]

37. Loyola-Vargas, V.M.; Ochoa-Alejo, N. An Introduction to Plant Tissue Culture: Advances and Perspectives. Methods Mol. Biol. 2018, 1815, 3-13.

38. Lu, C.; Vasil, V.; Vasil, I.K. Improved efficiency of somatic embryogenesis and plant regeneration in tissue cultures of maize (Zea mays L.). Theor. Appl. Genet. 1983, 66, 285-289. [CrossRef] [PubMed]

39. Sugimoto, K.; Temman, H.; Kadokura, S.; Matsunaga, S. To regenerate or not to regenerate: Factors that drive plant regeneration. Curr. Opin. Plant Biol. 2019, 47, 138-150. [CrossRef]

40. Han, Z.; Crisp, P.A.; Stelpflug, S.; Kaeppler, S.M.; Li, Q.; Springer, N.M. Heritable Epigenomic Changes to the Maize Methylome Resulting from Tissue Culture. Genetics 2018, 209, 983-995. [CrossRef]

41. Ranghoo-Sanmukhiya, V.M. Somaclonal Variation and Methods Used for Its Detection. In Propagation and Genetic Manipulation of Plants; Siddique, I., Ed.; Springer: Singapore, 2021; pp. 1-18.

42. Bidabadi, S.S.; Jain, S.M. Cellular, Molecular, and Physiological Aspects of In Vitro Plant Regeneration. Plants $2020,9,702$. [CrossRef]

43. Aguilera, A.; Garcia-Muse, T. Causes of genome instability. Annu. Rev. Genet. 2013, 47, 1-32. [CrossRef]

44. Knoll, A.; Fauser, F.; Puchta, H. DNA recombination in somatic plant cells: Mechanisms and evolutionary consequences. Chromosome Res. 2014, 22, 191-201. [CrossRef] [PubMed] 